\title{
Solutions to Yang-Mills Field Equations in Eight Dimensions and the Last Hopf Map
}

\author{
Bernard Grossman $^{1}$, Thomas W. Kephart ${ }^{2}$, and James D. Stasheff ${ }^{3}$ \\ 1 Physics Department, Rockefeller University, New York, NY 10021, USA \\ 2 Physics Department, Purdue University, West Lafayette, IN 47907, USA \\ 3 Mathematics Department, University of North Carolina, Chapel Hill, NC 27514, USA
}

\begin{abstract}
We will show that the Hopf map $S^{15} \stackrel{S^{7}}{\longrightarrow} S^{8}$ admits a sourceless, topologically non-trivial gauge field. This result will be cast in the form of a solution to eight dimensional Euclidean Yang-Mills field equations with topological charge $Q=1$. This solution is $\operatorname{Spin}(9)$ symmetric and leads to a new generalized duality condition $F \wedge F= \pm(F \wedge F)^{*}$.
\end{abstract}

It is becoming increasingly apparent that many of the subtleties of field theories can be understood only by investigating their global properties. This has necessarily led to the introduction of several new mathematical results and techniques into the physics literature, which has in turn prompted mathematicians in the areas of algebraic and differential topology to investigate a number of system of importance to physics. The most striking examples of this interdisciplinary pursuit are instanton solutions to the $D=4$ dimensional Yang-Mills field equations [1].

In this paper we will study the higher dimensional analog of the instanton, but first a few words of background are in order.

Trautman [2] was the first to realize that the one instanton solution to the $\mathrm{SU}(2) D=4$ Euclidean Yang-Mills field equations is related to a Hopf map [3]

$$
S^{7} \stackrel{S^{3}}{\longrightarrow} S^{4}
$$

i.e., the mapping associated with a principal fibre bundle structure with base space $S^{4}$ (the one point compactification of Euclidean four-space) and fibre $S U(2) \sim S^{3}$. The canonical connection on this bundle defines a self-dual or anti-self-dual curvature tensor $F_{\mu \nu}$.

Nowakowski and Trautman [2] consider more generally the natural connection on Stiefel bundles, and Laquer [13] considers the Yang-Mills functional associated with principal bundles over homogeneous spaces. 
There are four fundamental Hopf fibrations

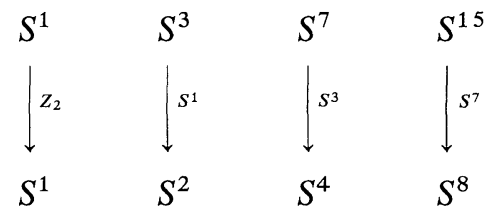

each one corresponding to one of the four normed division algebras [4], $R, C, H$, and $O$, the real, complex, quarternion, and octonion division algebras over the field of the reals.

Trautman [2] has observed that the first two Hopf maps can also describe physical systems. The first corresponds to the kink solution of the Sine-Gordon equation, and the second to the Dirac monopole of quantum-electrodynamics.

The fourth Hopf map allows a more exotic interpretation. Recently, Gürsey and Tze [5] have shown that it can be connected with the Freund-Rubin-Englert [6] $S^{7}$ spontaneous compactification of $N=1$ supergravity in eleven dimensions. Here we present an alternative viewpoint.

In the present paper we will study the properties of the last fundamental Hopf map (i.e. the Hopf fibration of the 15-sphere given above) in relation to solutions of pure eight dimensional Euclidean Yang-Mills field equations with gauge group Spin(8). We explicitly construct this solution and show that it has topological charge

$$
Q \propto \int(F \wedge F \wedge F \wedge F)
$$

with $Q= \pm 1$ for the appropriate choice of normalization. The solution is invariant under the action of Spin(9), the two fold covering of $O(9)$, which is a subgroup of the Euclidean conformal group $O(9,1)$ in eight dimensions. This result is analogous to the work of Jackiw and Rebbi [8] on the $D=4$ dimensional single instanton solution with gauge group $O(4)$. That solution was shown to have an $O(5)$ symmetry with $O(5) \subset O(5,1)$ (the $D=4$ Euclidean conformal group). Finally we show that the $D=8$ solution leads to a problem similar to the one studied by Atiyah et al. [9] of constructing general self dual gauge fields. Specifically, the $D=8$ case will be shown to satisfy a generalized duality. [ $\operatorname{In} D=4$ the $F= \pm F^{*}$ duality results from anti-self-dual solutions of the field equations. This is not the case in $D=8$. As we shall see, the generalized duality comes about algebraically.]

Let us proceed somewhat in analogy with Jackiw-Rebbi [8]. We consider a Spin(8) Euclidean Yang-Mills theory in 8-dimensions. This can be interpreted either as being a conformally invariant theory in $R^{8}$ with constraints at "infinity" or equivalently as an isometrically invariant theory on the unit sphere $S^{8}=\{r \mid r \cdot r=1\} \subset R^{9}$. Where appropriate, we write $r$ in terms of components $r_{a}$, $a=1, \ldots, 9$.

We seek a gauge potential (= connection) $\hat{A}=\left\{\hat{A}_{a}\right\}$ on the tangent bundle to $S^{8}$ so $\hat{A} \cdot r=0$ or $\hat{A}_{a} r_{a}=0$. We choose as our "ansatz"

$$
\hat{A}_{a}=i \alpha \Sigma_{a b} r_{b},
$$

where $\Sigma_{a b}$ is the irreducible 16-dimensional representation of Spin(9) and $\alpha$ is a constant to be determined. We can identify $R^{8}$ with the complement of the point 
$r_{9}=-1$ given by

$$
r_{\mu}=\frac{2 x_{\mu}}{1+x^{2}}, r_{9}=\frac{1-x^{2}}{1+x^{2}}
$$

where $\mu=1, \ldots, 8$ and $x^{2}=x_{\mu} x_{\mu}$.

We can use the $D=9$ dimensional angular momentum operator

$$
L_{a b}=-i r_{a} \frac{\partial}{\partial r_{b}}+i r_{b} \frac{\partial}{\partial r_{a}}
$$

to construct the third rank field strength tensor

$$
\hat{F}_{a b c}=i L_{a b} \hat{A}_{c}+r_{a}\left[\hat{A}_{b}, \hat{A}_{c}\right] \text {. }
$$

The Spin(9)-invariant action I is proportional to

$$
\int_{S^{8}} \operatorname{Tr} \hat{F}_{a b c} \hat{F}_{a b c} d \Omega \text {. }
$$

The "conventional" connection $A=\left\{A_{\mu}\right\}$ corresponding to $\hat{A}=\left\{A_{\alpha}\right\}$ is given by $\frac{1+x^{2}}{2} A_{\mu}=\hat{A}_{\mu}-x_{\mu} \hat{A}_{9}$. The corresponding field strength is then given as usual by

$$
F^{\mu \nu}=\partial^{\mu} A^{v}-\partial^{v} A^{\mu}+\left[A^{\mu}, A^{v}\right]
$$

or

$$
F=[\partial, A]+[A, A] .
$$

The above action $\mathrm{I}$ is then the same as (within normalization)

$$
\int_{R^{8}} \operatorname{Tr} F^{\mu \nu} F_{\mu \nu} d^{8}-x . \quad\left\|F^{\prime}\right\|^{2}
$$

The variational principle $\delta I=0$ then leads to the field equations

$$
i L_{a b} \hat{F}_{a b c}+r_{a}\left[\hat{A}_{b}, \hat{F}_{a b c}\right]-r_{b}\left[\hat{A}_{a}, \hat{F}_{a b c}\right]=0 .
$$

Substituting the ansatz (2) into the field equations gives

$$
14 i \alpha(\alpha+1)(\alpha+2) \Sigma_{a b} r_{b}=0
$$

with solutions $\alpha=0,-1,-2$. The solutions $\alpha=0$ and $\alpha=-2$ give $\hat{F}_{a b c}=0$; we turn to $\alpha=-1$.

Making a gauge transformation (= bundle automorphism)

$$
U=e^{i f\left(r_{9}\right)} \sum_{\mu 9} r_{\mu}
$$

we find

$$
A_{\mu}=\frac{-2 i \Sigma_{\mu} \mathcal{X}_{v}}{1+x^{2}}
$$

or

$$
A_{\mu}=\frac{-2 i \Sigma_{\mu \nu} X_{\nu}}{x^{2}\left(1+x^{2}\right)}
$$


for the respective choices

or

$$
f\left(r_{9}\right)=\frac{\cos ^{-1} r_{9}}{\left(1-r_{9}^{2}\right)^{1 / 2}}
$$

$$
f\left(r_{9}\right)=\frac{\cos ^{-1} r_{9}-\pi}{\left(1-r_{9}^{2}\right)^{1 / 2}} \text {. }
$$

Equation (14a) gives

$$
F_{\mu \nu}=\frac{4 \Sigma_{\mu \nu}}{\left(1+x^{2}\right)^{2}}
$$

while Eq. (14b) gives

$$
F_{\mu \nu}=\frac{4 \Sigma_{\mu \nu}}{x^{4}\left(1+x^{2}\right)^{2}} .
$$

Equations (2) through (15) are as in Jackiw-Rebbi with simple modifications.

For comparison, consider the natural connection, the Maurer-Cartan form $g^{-1} d g$, as a form on the tangent bundle to $\operatorname{Spin}(9)$. This form is left invariant. The irreducible 16-dimensional representation of Spin(9) composes with the MaurerCartan form to give a form right invariant under the subgroups $\operatorname{Spin}(7) \subset \operatorname{Spin}(8)$ so as to project to the coset spaces

$$
\begin{aligned}
& S^{15}=\operatorname{Spin}(9) / \operatorname{Spin}(7) \\
& \downarrow \\
& S^{8}=\operatorname{Spin}(9) / \operatorname{Spin}(8) .
\end{aligned}
$$

Expressed in terms of the coordinates $x=\left\{x_{\mu}\right\}$, the projected form is just the connection form $A=\left\{A_{\mu}\right\}$.

Regarding the field strength $F=\left\{F_{\mu \nu}\right\}$ as a curvature, we can regard

$$
P=N \int_{S^{8}}(F \wedge F \wedge F \wedge F) d V
$$

for a suitable normalization constant $N$, as computing the Euler number, or half the second Pontryagin number. For the Hopf fibration $S^{15} \rightarrow S^{8}$, this is known to be \pm 1 , since the classifying map of the fibration is a generator of $\pi_{7}(\operatorname{Spin}(8)) / \pi_{7}(\operatorname{Spin}(7)) \approx Z$. This then fixes the normalization in Eq. (17) uniquely and we identify half the Pontryagin number with the topological charge. Indeed, the integral in Eq. (17) is easily computed using the properties of the $\Sigma_{\mu \nu}$ (given below) and the form (15a) for $F_{\mu v}$. The spacial integral needed is

$$
\int_{S^{7}} d \Omega \cdot \int_{0}^{\infty} \frac{x^{7} d x}{\left(1+x^{2}\right)^{8}}=\frac{2 \pi^{4}}{\Gamma(4)} \cdot \frac{1}{280} .
$$

Now let us look more closely at the spinor representation of $\operatorname{Spin}(n)$. The $16 \times 16 \operatorname{Spin}(9)$ irreducible representation matrices $\left\{\Sigma_{a b}\right\}$ decompose via a $\frac{1}{2}\left(1 \pm \gamma_{9}\right)$ projection into the two irreducible $8 \times 8$ spinor representations $\left\{\Sigma_{\mu \nu}\right\}$ and $\left\{\bar{\Sigma}_{\mu \nu}\right\}$ of Spin(8). A useful choice for the $\left\{\Sigma_{\mu v}\right\}$ matrices is the following [10]

$$
\Sigma_{\mu \nu}=\Sigma_{A B} \oplus \Gamma_{A} ; \quad A=1,2, \ldots, 7,
$$


where

$$
\Sigma_{A B} \equiv(1 / 2 i)\left[\Gamma_{A}, \Gamma_{B}\right]
$$

and the $\Gamma$ 's are defined by

$$
\begin{aligned}
& \Gamma_{1}=-\sigma_{112} ; \Gamma_{2}=-\sigma_{120} ; \Gamma_{3}=\sigma_{132} ; \Gamma_{4}=\sigma_{221} ; \\
& \Gamma_{5}=-\sigma_{220} ; \Gamma_{6}=\sigma_{202} ; \Gamma_{7}=-\sigma_{300} .
\end{aligned}
$$

Here we have defined

$$
\sigma_{i j k}=\sigma_{i} \otimes \sigma_{j} \otimes \sigma_{k}
$$

with $\sigma_{1}, \sigma_{2}$, and $\sigma_{3}$ the $2 \times 2$ Pauli matrices and $\sigma_{0}$ the $2 \times 2$ unit matrix.

A product can be defined on the seven $\Gamma$ 's such that they form a representation of the nonreal octonians [10]. Furthermore, the group element $g$ as well as the Maurer-Cartan form $g^{-1} d g$ can be given an octonionic interpretation. To accomplish this, we identify the map of the octonionic $S^{7}$ onto the $S^{7}$ at infinity in $D=8$ dimensional Euclidean space with

$$
g=\frac{1 \cdot x_{0}+i \Gamma_{A} x_{A}}{|x|} .
$$

This is in direct analogy with the 4-dimensional case where

$$
g=\frac{1 \cdot x_{0}+i \boldsymbol{\sigma} \cdot \mathbf{x}}{|x|}
$$

with the Pauli matrices $\sigma$ seen as a representation of the nonreal quaternions.

Let us now summarize several more useful identities satisfied by the $\Sigma$ 's and $\Gamma$ 's $[11,12]$ :

The $\Gamma$ 's satisfy (indices in square brackets are to be antisymmetrized)

$$
\Gamma_{[A} \Gamma_{B} \Gamma_{C} \Gamma_{D]}=\frac{1}{4 !} \varepsilon_{A B C D E F G} \Gamma_{E} \Gamma_{F} \Gamma_{G}
$$

thus

$$
\Sigma_{8[A} \Sigma_{B C]}=\frac{1}{4 !} \varepsilon_{8 A B C D E F G} \Sigma_{D E} \Sigma_{F G}
$$

and so

$$
\Sigma_{[\mu \nu} \Sigma_{\varrho \sigma]}=\frac{1}{4 !} \varepsilon_{\mu \nu \varrho \sigma \alpha \beta \gamma \delta} \Sigma_{\alpha \beta} \Sigma_{\gamma \delta}
$$

Therefore we find that, purely as a consequence of the properties of the $\Sigma$ 's, the $D=8$ field strengths $F=\left\{F_{\mu v}\right\}$ of Eq. (15) satisfy the duality equation

$$
F \wedge F= \pm(F \wedge F)^{*},
$$

where the $(+)$ and $(-)$ correspond to self-dual and antiselfdual representations $\Sigma$ and $\bar{\Sigma}$, respectively.

One final identity satisfied by the $\Sigma$ 's and needed to complete the evaluation of the normalization in Eq. (17) is, using Eqs. (19)-(22),

$$
\operatorname{Tr} \Sigma_{12} \Sigma_{34} \Sigma_{56} \Sigma_{78}=8 .
$$


For the sake of completeness let us make contact with Ref. [5]. Fixing one index ( $\mu$ say) at 8 in Eq. (28) forces the remaining indices to run from 1 to 7 so we write $v, \varrho, \ldots$, as $A, B, \ldots$ such that

$$
F_{8[A} F_{B C]}=\frac{1}{4 !} \varepsilon_{8 A B C D E F G} F_{D E} F_{F G} .
$$

Defining $\Psi$ and $\Phi$ by

$$
\left(\frac{2}{1+x^{2}}\right)^{4} \Psi_{A B C}=F_{8[A} F_{B C]}
$$

and

$$
\left(\frac{2}{1+x^{2}}\right)^{4} \Phi_{E F G H}=F_{[E F} F_{G H]},
$$

we have

$$
\Psi_{A B C}=\frac{1}{4 !} \varepsilon_{A B C D E F G} \Phi_{D E F G} .
$$

But this is quite similar to the duality condition described by Gürsey and Tze [5] when $A, B$, and $C$ are restricted to the seven octonionic triples i.e., for pure nonreal unit octonions $e_{i}$

$$
e_{i} e_{j}=\delta_{i j}+\psi_{i j k} e_{k},
$$

where

$$
i j k=(123,246,435,367,651,572,714) .
$$

As a consequence of triality, $\operatorname{Spin}(8)$ has three 35-dimensional inequivalent irreducible representations. Under the reduction $\operatorname{Spin}(8) \rightarrow \operatorname{Spin}(7)$, two of the (35) $s$ remain irreducible while the third $(\underset{2}{3}) \rightarrow(1)+(7)+(27)$. On further reduction to $G_{2}$, all three of the (35)s of $\operatorname{Spin}(8)$ reduce to $(\underset{\sim}{1})+(7)+(27)$.

The combination of generators $\Sigma_{[\mu \nu} \Sigma_{\rho \sigma]}$ that enters Eq. (28) transforms as a (252) of Spin(8). The projections of $(\underset{\sim}{35}) s$ to $(\underset{\sim}{1})+(7)+(27)$ can be accomplished by a straightforward application of the properties of the restricted tensors $\Phi_{i j k l}$ and $\Psi_{i j k}$. Though we will not pursue this further here, detailed formulae are provided in Ref. [12].

In $D=4$ the condition $F= \pm F^{*}$ leads to a set of linear equations which can be solved to construct general self-dual Yang-Mills gauge fields. For $D=8$ the conditions are more complicated, but a similar problem of constructing solutions to the Yang-Mills field equations with a "generalized restricted duality" occurs, cf., Eq. (28). It is useful to note in this regard that there is a complete analogy between the homotopy groups relevant to the $D=8$ solutions,

$$
\pi_{7}(\operatorname{Spin}(8))=Z+Z, \pi_{7}(\operatorname{Spin}(7))=Z \text { and } \pi_{7}\left(G_{2}\right)=1
$$

with those relevant to the $D=4$ instantons

$$
\pi_{3}(O(4))=Z+Z, \pi_{3}(\mathrm{SU}(2))=Z \quad \text { and } \quad \pi_{3}(1)=1 .
$$


As a final remark we observe that the field strength of Eq. (15a) has action

$$
\int F_{\mu \nu} F_{\mu \nu} d^{8} x \sim \int \frac{x^{7} d x}{\left(1+x^{2}\right)^{4}} \sim \int \frac{d x}{x},
$$

which is logarithmically divergent. This is somewhat discouraging; however $D=8$ Yang-Mills theories are inherently nonrenormalizable and the fact that the divergence is only logarithmic gives one hope that it may be controllable if the $D=8$ Yang-Mills theory is properly embedded in some well behaved higher $(D \geqq 8)$ dimensional theory.

Acknowledgements. Two of us (B.G. and T.K.) thank Arthur Halprin and the Lewes Center for Physics for hospitality while this work was in progress. The research of B.G. was supported by U.S. DOE grant No. DE-AC02-82ER40033.B000, that of T.K. by DOE grant No. DE-AC02-76ER-01428, and that of J.S. by NSF grant No. MCS-790340.

\section{References}

1. Belavin, A.A., Polyakov, A.M., Schwarz, A.S., Tyupkin, Yu.S.: Pseudoparticle solutions of the Yang-Mills equations. Phys. Lett. 59 B, 85 (1975)

2. Trautman, A.: Int. J. Theor. Phys. 16, 561 (1977);

Nowakowski, J., Trautman, A.: Natural connections on Stiefel bundles are sourceless gauge fields. J. Math. Phys. 19, 1100 (1978)

3. Hopf, H.: Über die Abbildungen Sphären auf Sphären niedriger Dimension. Fund. Math. 25, 427-440 (1935)

Steenrod, N.: The topology of fibre bundles. Princeton NJ: Princeton University Press 1951

4. Porteous, I.R.: Topological geometry (2nd edn.). Cambridge, UK: Cambridge University Press 1981

5. Gürsey, F., Tze, C.-H.: Octonionic torsion on $S^{7}$ and Englert's compactification of $d=11$ supergravity. Phys. Lett. 127B, 191 (1983)

6. Freund, P.G.O., Rubin, M.: Dynamics of dimensional reduction. Phys. Lett. 97 B, 233 (1980) Englert, F.: Spontaneous compactification of eleven-dimensional supergravity. Phys. Lett. 119B, 339 (1982)

7. Milnor, J., Stasheff, J.: Characteristic classes. Princeton, NJ : Princeton University Press 1974

8. Jackiw, R., Rebbi, C.: Conformal properties of a Yang-Mills pseudoparticle. Phys. Rev. D 14, 517 (1976)

9. Atiyah, M.F., Hitchen, N.J., Drinfeld, V.G., Manin, Yu.I.: Construction of instantons. Phys. Lett. 65A, 185 (1978)

10. Günaydin, M., Gürsey, F.: Quark structure and octonions. J. Math. Phys. 14, 1651 (1973)

11. Cremmer, E., Julia, B.: The SO (8) supergravity. Nucl. Phys. B 159, 141 (1979)

12. deWit, B., Nicolai, H.: The parallelizing $S^{7}$ torsion in gauged $N=8$ supergravity. Nucl. Phys. B 231, 506 (1984)

13. Laquer, T.: Mich. Math. J. (to appear)

Communicated by A. Jaffe

Received March 19, 1984; in revised form May 29, 1984

Note added in proof: In fact, the action is finite when the theory is defined on $S^{8}$. We would like to thank Roman Jackiw for pointing this out. 
